\title{
Author Index to Volume 36
}

\author{
Abman SH, 402 \\ Abraham M, 545 \\ Abrams SA, 424 \\ Act JA, 49 \\ Agata Y, 805 \\ Aldridge RF, 699 \\ Alfthan $\mathrm{H}, 221$ \\ Alves C, 749 \\ Andersson S, 55 \\ Andrews NC, 419 \\ Ank BJ, 792 \\ Anttila R, 745 \\ Armitage MM, 414 \\ Artwohl J, 660 \\ Bach TA, 144 \\ Backeljauw PF, 749 \\ Bagchi A, 244 \\ Banerjee A, 474 \\ Bang P, 152, 528 \\ Barbato R, 182 \\ Barbera C, 347 \\ Bard H, 253 \\ Belik J, 34 \\ Benelli C, 707 \\ Benson LN, 49, 373 \\ Bent RC, 494 \\ Berger JI, 776 \\ Bergman AJIW, 582 \\ Bernard A, 771 \\ Bernard F, 175 \\ Bernasconi S, 654 \\ Berry GT, 613 \\ Bestetti GE, 315 \\ Beyer EC, 561 \\ Bifano EM, 90 \\ Binder ND, 102 \\ Blachier F, 175 \\ Blum WF, 528 \\ Boesiger P, 436 \\ Bolin K, 660 \\ Bonino F, 347 \\ Bracci R, 619 \\ Brinkmann AO, 227 \\ Broerse HM, 578 \\ Brommer EJP, 257 \\ Bronner F, 424 \\ Brouwer A, 468 \\ Brown G, 699 \\ Brown RK, 487 \\ Brubakk A-M, 334 \\ Brun NC, 20 \\ Brunetto MR, 347 \\ Brus F, 647 \\ Buchli R, 436 \\ Bueva A, 572 \\ Bührer C, 799 \\ Buonocore G, 619 \\ Burlina AB, 182 \\ Burton KA, 77 \\ Buschang PH, 265
}

Buts J-P, 522

Cady EB, 699

Calvo P, 347

Caniglia M, 60

Carey HV, 144

Carver T, 293

Caspi J, 49

Caughy MO, 207

Cella SG, 169, 315

Chen S, 561

Cherouvrier F, 816

Chess PR, 481

Chrousos GP, 215

Ciccimarra F, 60

Cleveland W, 749

Coles JG, 49

Colomb V, 202

Colonna VDG, 315

Cooper CE, 699

Coscia A, 347

Costeloe K, 364

Cox C, 122, 358

Cox D, 763

Curstedt T, 784

Cusson RM, 207

Dagnelie PC, 194

Dalemans W, 137

Darcy-Vrillon B, 175

Darmaun D, 202

Dastoli G, 347

Dauncey MJ, 77

Davenport ML, 749

David-Cu R, 283

Davidson F, 351

Davidson TL, 501

Davidsson L, 816

Davis JM, 494

Davis LM, 561

de Blic J, 667

De Filippo M, 619

de Groot R, 578

De Haan HH, 595

De Haan J, 595

de Jong RCJW, 323

De Keyser N, 522

De Meirleir L, 707

de Muinck Keizer-Schrama SMPF, 323

De Praeter C, 64

De Raedemaeker L, 522

de Ridder MAJ, 323

De Tullio R, 182

DeCristofaro JD, 719

Defoort P, 71

Degenhart HJ, 227

Deka R, 414

DeKing D, 358

Delaisi B, 667

DeLong DJ, 358

\author{
DeLozier-Blanchet C, 441 \\ Delpy DT, 699 \\ deMello DE, 380 \\ Demir A, 221 \\ Denne SC, 429 \\ Desguerre I, 707 \\ Devarajan P, 129 \\ Devaskar UP, 380 \\ Dhont M, 64 \\ Di Lisa F, 182 \\ DiPietro JA, 207 \\ Dokianakis SG, 732, 738 \\ Donckerwolcke RA, 323 \\ Donckerwolcke RAMG, 582 \\ Donovan SM, 159 \\ Dracker RA, 90 \\ Drop SLS, 227, 323 \\ Duchan R, 545 \\ Dudenhausen JW, 799 \\ Duée P-H, 175 \\ Duquesnoy P, 654 \\ Duran M, 582 \\ Edwards AD, 699 \\ Eibl MM, 235 \\ Eidson M, 749 \\ Eisen R, 456 \\ Ensor JE, 244 \\ Ewald U, 550 \\ Extermann P, 441
}

Faber JJ, 102

Falkner B, 95

Fauler J, 449

Fedderson JA, 755

Fernández-Ulloa M, 329

Ferrell RE, 414

Fewell JE, 501

Finegold DN, 414

Fineman JR, 394

Finkelstein JN, 481

Fischer MB, 235

Fleming PJ, 713

Fox NA, 207

Frahm J, 409

Freund MM, 771

Frölich JC, 449

Fujino N, 805

Gagnon C, 253

Gaillard D, 137

Galan P, 816

Galiani M, 414

Gappa M, 364

Gewolb IH, 115

Ghizzoni L, 654

Gibson JB, 613

Gibson KJ, 506

Gibson RL, 776

Gioia D, 619

Gitzelmann R, 436

Goldstein B, 358
Goossens M, 654

Gospe Jr SM, 811

Goulet O, 202

Govaert P, 71

Govindarajan R, 380

Graulich J, 799

Greisen G, 20

Grilli R, 169

Guignard J-P, 572

Gupta S, 115

Gustafsson J, 550

Guthrie RD, 732, 738

Haaland K, 601

Hagenfeldt L, 111

Halley DJJ, 227

Halliday D, 713

Hallman M, 55, 387

Han RNN, 763

Hanefeld F, 409

Hänicke W, 409

Hankard R, 202

Harvey C, 514

Hasaart THM, 595

Hasday JD, 244

Hay Jr WW, 293

Hayek A, 537

Heikinheimo M, 85

Heinonen OJ, 288

Helms G, 409

Henry MM, 724

Hercberg S, 816

Herman SL, 49

Herting E, 784

Heymann MA, 474

Heymen S, 380

Hinnrasky J, 137

Hiraishi S, 805

Hirota H, 805

Hiura K, 805

Hodgins MB, 227

Hokken-Koelega ACS, 323

Holzbach U, 409

Hoogenboezem T, 227

Hop WCJ, 578

Horiguchi Y, 805

Hostetter MK, 692

Houston K, 265

Hull AD, 25

Hulman S, 95

Hurrell RF, 816

Iannaccone ST, 265

Ierardi JA, 642

Ijlst L, 111

Ijzermans ACM, 595

Ito $\mathrm{M}, 340$

Ivy $\mathrm{DD}, 402$

Iwamoto K, 660

Jacobson LJ, 293

Jadhav LS, 300 
Jarstrand C, 784

Jaswon MS, 623

Jean $G, 628$

Jeffries S, 215

Jiménez-Flores R, 159

Jones HM, 623

Juillerat MA, 816

Kamath SG, 461

Kari MA, 387

Karlsson B, 601

Karn CA, 429

Kastenmayer P, 816

Katz BZ, 456

Kelly FJ, 487

Kemp JS, 7

Kempski MH, 358

Keppler D, 449

Kinsella JP, 402

Kint J, 64, 71

Kirkbride V, 699

Kivivuori SM, 85

Klimah P, 660

Koistinen H, 745

Koistinen R, 745

Konduri GG, 41

Koopman-Esseboom C, 468

Koppel R, 763

Koskinen T, 607

Krug-Wispé S, 329

Kuroda Y, 340

Laborde K, 667

Lachance C, 253

Ladinser B, 635

LaGamma EF, 719

Laird MR, 102

Lallemand A, 137

Lane AT, 306

Langhendries J-P, 771

Lauwerys R, 771

Leffler CW, 589

Leigh T, 545

Leitch CA, 429

Lenoir G, 667

Leroy J, 64, 71

Lewinsky RM, 373

Liebaers I, 707

Liechty EA, 429

Linch DC, 623

Lindemans J, 578

Linker DT, 334

Lissens W, 107

Liu YM, 429

Locatelli V, 169, 315

Long DM, 25

Lopez AD, 537

Lorah K, 90

Lorek A, 699

Lumbers ER, 506

Lutkeschipholt IJ, 468

Madison KC, 306

Mally MI, 537

Mancini AJ, 306

Manco-Johnson MJ, 293

Marquardt I, 409

Marsac C, 707

Martinussen M, 334

Mason JI, 660

Matise TC, 414
Mayatepek E, 449

Mayock DE, 776

McCrea KA, 244

McIntosh N, 351

McNamara JG, 456

McNeil LK, 159

Meier D, 436

Menabŏ R, 182

Mencia-Huerta JM, 628

Menon G, 351

Merritt TA, 494

Meslin J-C, 175

Mimouni F, 329

Misawa H, 805

Monnens LAH, 257

Morel M-T, 175

Morris Jr JG, 115

Morse DC, 468

Mousson B, 582

Müller EE, 169, 315

Naito E, 340

Namgung R, 329

Nelson C, 12

Nelson VE, 7

Niaudet P, 628

Nikali K, 607

Nowatari M, 805

Obladen M, 779

Odle J, 159

Oetomo SB, 647

Oguchi K, 805

Oh SY, 300

Okken A, 647

Oosterwijk JC, 227

Otonkoski T, 537

Owen-Reece H, 699

Page WV, 755

Palit A, 90

Palmieri MJ, 613

Palumbo A, 60

Pandian MR, 414

Pang S, 215, 660

Panigrahi P, 115

Parkman HP, 642

Paul DA, 642

Peabody SS, 494

Pearce WJ, 25

Peebles D, 699

Peltonen L, 607

Penn JS, 724

Penrice J, 699

Perlmutter DH, 271

Perry YM, 414

Perugini L, 347

Petell JK, 635

Phelps DL, 122

Picciolini E, 619

Pihko H, 607

Pintos-Morell G, 628

Pitkánen O, 55

Pittschieler K, 635

Poggi F, 707

Poll-The BT, 582

Ponsot G, 707

Poorthuis BJHM, 187

Porter CC, 755

Posho L, 175

Puchelle E, 137
Rabbette PS, 364

Rabinovitch M, 763

Raghunath M, 441

Raivio KO, 387

Rakotoambinina B, 667

Randone A, 347

Rasool O, 784

Redding GJ, 776

Refsum H, 194

Relman DA, 567

Rengs H, 235

Requardt M, 409

Reynolds EOR, 699

Reynolds RA, 613

Ricour C, 202

Riddick L, 215

Ris-Stalpers C, 227

Rischák K, 278

Ritchie JWK, 373

Robert J-J, 667

Roberts RL, 792

Robertson B, 784

Robillard JE, 755

Rodriguez D, 707

Rogerson FM, 660

Roman C, 474

Romano G, 60

Romme AE, 187

Rongier M, 202

Rosenfeld RG, 152, 528

Rossi GL, 315

Roth SC, 699

Rousset M, 514

Rowley AH, 567

Rudolph AM, 687

Ruocco S, 137

Ryan JP, 642

Ryan RM, 481

Saeed DB, 811

Saffitz JE, 561

Saini U, 456

Salamino F, 182

Salem P, 628

Salimi B, 456

Sambol SP, 567

Sanders RJ, 122

Santoro P, 60

Saudubray J-M, 707

Sauer PJJ, 468, 578

Schanler RJ, 424

Scher MS, 732, 738

Schlesinger MJ, 1

Schneede J, 194

Schutte BC, 755

Schwander J, 528

Schwarz H-P, 235

Sclabassi RJ, 738

Seale NS, 265

Segal S, 613

Segar JL, 755

Seppälä M, 745

Shibata M, 589

Shortland GJ, 713

Shulman ST, 567

Siegel NJ, 129

Siimes MA, 85, 745

Siliprandi N, 182

Silly C, 667

Silverstein FS, 12
Sinkin RA, 122

Skovlund E, 601

Slayter MV, 494

Sleddens HFBM, 227

Smeitink JAM, 582

Smets K, 71

Smith CH, 461

Smoller BR, 306

Soifer SJ, 394

Sookdeo-Drost S, 306

Sosenko IRS, 380

Southall DP, 364

Standaert TA, 776

Stangenberg M, 528

States B, 613

Steinmann B, 436, 441

Stenman U-H, 221

Steppe DA, 732, 738

Stibenz D, 799

Stiehm ER, 792

Stijnen T, 323

StÖckler S, 409

Stocks J, 364

Sullivan J, 567

Sun B, 784

Sun M, 738

Sunehag A, 550

Suomalainen A, 607

Superti-Furga A, 441

Supnet MC, 283

Swallow DM, 514

Swart P, 660

Szollár L, 278

Szwarc RS, 373

Taciak V, 244

Takala J, 288

Takeda E, 340

Takei Y, 699

Tanswell AK, 763

Tapanainen PJ, 152

Teppo A-M, 85

Terai M, 567

Thach BT, 7

Thielemans N, 771

Thoresen M, 601

Thorkelsson T, 329

Tivey DR, 77

Tolman BL, 724

Tomsits E, 278

Torresani T, 654

Torsello A, 169, 315

Townsend SF, 293

Trapman J, 227

Truog WE, 776

Tsang RC, 329

Tsikas D, 449

Tuinstra LGMT, 468

Ueland PM, 194

Underwood LE, 749

Unterman TG, 152

Vaisman N, 545

Van Belle H, 595

van Bergen W, 323

van de Kar NCAJ, 257

van den Anker JN, 578

van der Heijden, 578

Van der Paauw CG, 468

van Hinsbergh VWM, 257 
Van Kets H, 71

Van Leìrde M, 771

van Oeveren W, 647

van Staveren WA, 194

van Zaal MAE, 323

Vanderford PA, 394

Vanhaesebrouck P, 64, 71

Varsila E, 55

Venge P, 387

Venizelos $N, 111$

Verleun-Mooijman MCT, 227

Vianey-Saban C, 582

Vieira NE, 424

Viggiano D, 60

Vik T, 334
Viscardi RM, 244

Voet $\mathrm{H}, 545$

Vogel E, 235

Vollset SE, 194

Vottero A, 654

Voutilainen R, 221

Vreman HJ, 152

Wagemaker G, 187

Walter JH, 713

Walther FJ, 283

Wanders RJA, 111

Wang Y, 514

Wehrenberg WB, 169, 315

Weisglas-Kuperus N, 468
Westerterp K, 545

Westgren M, 528

Westphale EM, 561

White CR, 25

Why SV, 129

Widness JA, 253

Willemsen R, 187

Williams DA, 557

Williams JR, 351

Wilson GJ, 49

Wilson K, 152

Wiswell TE, 494

Wolf HM, 235

Wolff ED, 323
Wolinsky SM, 567

Wong J, 394

Woolf PD, 358

Wyatt JS, 699

Wylezinska M, 699

Yakubu MA, 589

Yang X, 660

Yao AC, 334

Yergey AL, 424

Yokota I, 340

Yoshijima S, 340

Zeman FJ, 811

Zhou SS, 811 